East African Medical Journal Vol. 81 No. 10 October 2004

CYSTOSARCOMA PHYLLODES: REPORT OF TWO CASES

M. H. Dahniya, FWACS (Rad), FRCR, Department of Radiology, Fujairah Hospital, Fujairah, United Arab Emirates, R. M. Hanna, MD, FRCR, Department of Diagnostic Radiology and Imaging, Al-Sabah Hospital, Kuwait and S. D. Ashebu, MBBS, FWACS, (Rad), Department of Radiology, Al-Adan Hospital, Kuwait

Request for reprints to: Dr. S. D. Ashebu, P.O. Box 4372132052 Hawally. Kuwait

\title{
CYSTOSARCOMA PHYLLODES: REPORT OF TWO CASES
}

\section{H. DAHNIYA, R. M. HANNA and S. D. ASHEBU}

\section{SUMMARY}

\begin{abstract}
Cystosarcoma phyllodes is a rare predominantly benign tumour usually affecting the female breast. In a retrospective review of giant breast masses seen over a period of 20 years, we encountered two cases of this tumour, one benign and the other malignant, the latter exhibiting the very unusual feature of axially Iymph node metastases. We present these two cases and briefly review the literature.
\end{abstract}

\section{INTRODUCTION}

Cystosarcoma phyllodes (CSP) is a fibroepithelial neoplasm that histologically resembles a fibroadenoma, but unlike the latter, it has a high incidence of local recurrence after surgery and a potential to metastasize. It is rare especially in adolescence. Distinction between fibroadenoma, benign CSP and malignant CSP may be difficult. Treatment is, in the majority of the cases, by complete excision and continued follow-up.

\section{CASE REPORTS}

Case 1: A 40 year old Arab woman presented with a rapidly growing painless mass in the upper half of her right breast for a period of one month. The mass was mobile and unattached to skin. There was no axillary lymphadenopathy. Physical examination apart from the breast mass was normal. Chest X-ray and laboratory tests were also normal. Mammography revealed a well-defined, dense mass measuring 9 $98 \times 8 \mathrm{~cm}$ (Figure 1). On ultrasonography, it was well-defined and hypoechoic with small cystic foc1. A giant fibroadenoma was diagnosed and FNAB was considered unnecessary. The mass was excised and pathological examination showed features of benign phyllodes tumour.

Case 2: The patient was a 40 ycar old Arab woman who presented with a rapidly growing, painless right breast mass over a period of five months. Physical examination revealed right axillary lymphadenopathy. Chest X-ray and laboratory tests were normal. Mammography showed a large. dense and predominantly well-defined mass measuring $15 \times 15 \times 15 \mathrm{~cm}$ with macrocalcification. Part of its margin was ill-defined (Figure 2). There were enlarged dense right axillary lymph nodes and the overlying skin showing dilated veins. Since a malignant lesion could not be excluded clinically, a right mastectomy with clearance of the axilla was performed. Pathological examination of both the mass and lymph nodes showed malignant phyllodes tumour.
Figure 1

Mammogram of a 40 year old woman with a rapidly growing mass in her right breast showing a giant welldefined mass of soft tissue density displacing normal breast tissue. It was excised and pathological examination showed benign phyllodes tumour

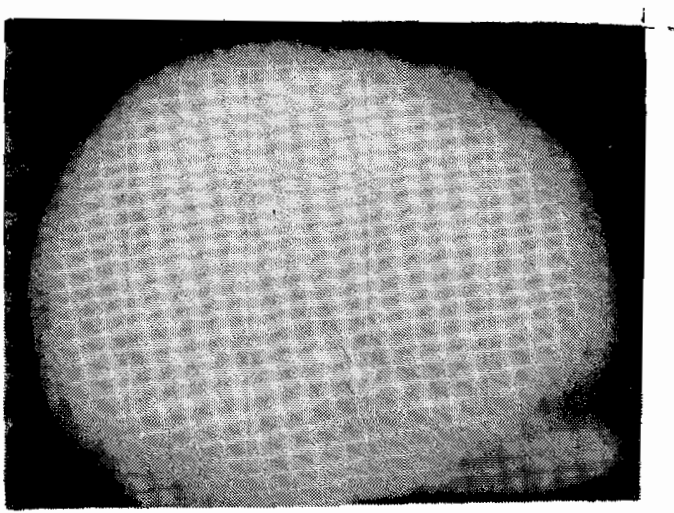

Figure 2

Mammogram of a 40 year old woman with a rapidly growing right breast mass showing a giant soft tissue mass with an ill-defined posterior margin and macrocalcification (arrows). Enlarged and dense lvmph nodes were demonstrated in the axilla. Pathological examination after excision showed malignani phyllodes tumour of the breast and lymph nodes

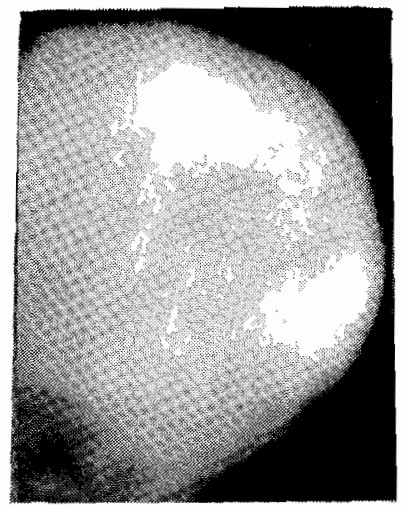




\section{DISCUSSION}

Cytosarcoma phyllodes (CSP) is a rare mixed fibroepithelial tumour that histologically resembles a fibroadenoma(1). Most cases are reported in women aged 30 to 70 years, with the majority encountered in the fifth decade(2). In adolescent females, the average age of occurrence is 15 years( 3 ). Out of 25 adolescent females with this tumour, seven occurred below the age of 15 years, eight between 15 and 17 years and 10 between 18 and 20 years(3). Our patients were both 40 years old.

Most phyllodes tumours present as rapidly enlarging breast masses. On physical examination, they are usually large, sometimes massive $(5-10 \mathrm{~cm})(4)$ and may deform the breast. Inspite of their size, they usually remain mobile and not fixed to the chest wall. They are often smooth on palpation, but some have an unevenly bosselated surface. Occasionally, ulceration of the overlying skin recurs due to pressure necrosis. The axillary lymph nodes are usually not enlarged and the nipple-areolar complex are rarely involved. In the absence of metastatic disease, physical findings in the cardiovascular, respiratory and other systems are usually normal. Although most are benign, only half are encapsulated and may recur if incompletely excised(4). The recurrence rates are $2.7 \%$ for benign tumours and $33 \%$ for malignant tumours in adolescent females(5). In adults, the average rate of recurrence is estimated at 14 to $20 \%$ for benign tumours, $27 \%$ for malignant tumours $(6,7)$. Patients with metastases may present with syr: ptoms such as dyspnoea, fatigue and bone pain.

The actual incidence of malignancy in these tumours is unknown. Metastases are almost always haematogenous. The tumour may spread locally or metastasize to the lungs, mediastinum and the skeleton. Lymphatic spread to the axillary lymph nodes are very rare(8). Unusual metastatic disease reported include giant metastasis to the lung(9), and metastasis to the groin(10). A giant malignant CSP of the breast associated with hypoglycaemia has also been reported(11). Pleomorphic differentiation of CSP into other specialised stroma such as liposarcoma and fibrosarcoma, osteosarcoma can occur(12).

The characteristic mammographic finding is a round, oval or slightly lobulated high density mass with well-defined margins and no calcification $(6,13,14)$. The most common sonographic features are lobulated shape, smooth margins and heterogeneous internal echoes without acoustic attenuation. Some tumours show round or oval shape, echogenic rim, homogeneous internal echoes or cystic spaces $(8,15)$.

Mammography and ultrasonography, while important in the diagnosis of breast diseases, are unreliable in differentiating benign CSP from fibroadenomas and malignant CSP.
MR imaging features of phyllodes tumour reported include oval or lobulated shape with internal septation, high signal intensity on $\mathrm{T} 2$ weighted images and rapid contrast enhancement on dynamic imaging(1), but dynamic enhancement pattern does not distinguish between the benign and malignant variety of this or other breast tumours $(16,17)$.

Histologically, CSP is classified into three categories: benign, borderline (intermediate), and malignant based on stromal hyperplasia(3). A combination of mitotic rate, stromal cellularity and atypia, presence of infiltrating borders and presence or absence of stromal growth, are the criteria used to distinguish between benign and malignant CSP. Stromal growth is the most important histological criterion of malignancy(18). However, distinguishing between fibroadenoma, benign CSP and malignant CSP may be most difficult even for the most experienced pathologist.

The poor correlation between the histological appearance and the clinical behaviour of CSP has made evolution of its surgical management difficult, but because most are benign, the general trend now is to do a wide surgical incision rather than mastectomy as the initial treatment $(15,19)$. Less extensive resections are recommended $(20,21)$. In one series, out of 20 patients treated by initial wide excision, 15 had no further recurrences. Local recurrence in the remaining five patients was treated by repeat excision or mastectomy (19). Local recurrence does not imply associated systemic spread. Some patients with very large tumours or suspicion of malignancy may be treated primarily by mastectomy, as in our second patient.

Local recurrence after mastectomy require a wide excision of the recurrence, including a margin of the underlying pectoralis muscle, if it is attached to the tumour(19). Deep recurrence may require aggressive excision of the involved thoracic wall with subsequent reconstruction(22). Treatment of systemic disease by chemotherapy or radiotherapy is sometimes necessary but has rarely resulted in any long term survival(23). The clinical and radiological diagnosis in our first patient was giant fibroadenoma and FNAB was therefore regarded as unnecessary. The tumour was excised and confirmed histologically as CSP. There has been no recurrence during a follow up period of six years.

Certain mammographic features observed in our second patient: ill definition of part of the outline of the mass, distended veins and axillary lymphadenopathy were suspicious of malignancy. Mastectomy and axillary clearance were therefore considered necessary. Histology of the mass and lymph nodes confirmed malignant phyllodes tumour. The axillary metastatic lymphadenopathy is a very unusual occurrence in malignant CSP, since lymphatic spread is uncommon(8). The patient had no recurrence for two years, but has been lost to follow-up. 


\section{REFERENCES}

1. Farria, D.M., Gorczyca, D.P., Barsky, S.H., Sinha, S. and Bassett, L.W. Benign phyllodes tumor of the breast: MR imaging features. Amer. J. Radio. 1996; 167:187-189.

2. Johnson, R.L., Page, D.L. and Anderson, T.J. Sarcomas of the breast. In: Page D.L. Anderson T.J. Eds. Diagnostic histopathology of the breast. New York, Churchill Livingston. 1987; 335-353 .

3. Nambiar, R. and Kutty, M.K., Giant fibroadenoma, (Cystosarcoma phyllodes) in adolescent females - a clinicopathological study. Brit. J. Surg. 1974; 61:113-117.

4. Evans, W.P. Breast masses. Radio. Clin. North. Amer. 1995; 13:1088

5. Briggs, R.M., Walters, M. and Rosenthal, D. Cystosarcoma phyllodes in adolescent female patients. Amer. J. Surg. 1983; 146:712-714.

6. Page. J.E. and Williams, J.E. The radiological features of phyllodes tumour of the breast with clinicopathological correlation. Clin. Radiol. 1991; 44:8-12.

7. Petruszka. M. and Barnes, L. Cystosarcoma phyllodes: a clinicopathological analysis of 42 cases. Cancer. 1978: 41:1974-1983

8. Reinfuss, M., Mitus, J., Smolak, K, and Stelmach, A. Malignant phyllodes tumors of the breast: a clinical and pathological analysis of 55 cases. Euro. I Cancer. 1993; 29A: $1252-1256$.

9. Takahashi, M., Murata, K., Mori, M. et al. Giant metastatic cystosarcoma phyllodes to the lung: $\mathrm{CT}$ and $\mathrm{MR}$ findings. Radiat. Med. 1992; 10:210-213.

10. Singh. J., Singh, B. and Chander, J. Extramammary cystosarcoma phyllodes with bilateral breast involvement. Aust. N. Z. J. Surg. 1980; 50:56-58

11 Kataoka, T., Haruta, R.. Goto, T. et al. Malignant phyllodes tumor of the breast with hypoglycaemia: report of a case. Jpn .J. Clin. Oncol. 1998; 28:276-280
12. Jimenez, J.F., Gloster, E.S. and Perrot, L.T. Liposarcoma arising within a cystosarcoma phyllodes. J. Surg. Oncol. 1986; 31:294-298.

13. D'Orsi, C.J., Feldhaus, I. and Sonnenfeld, M. Unusual lesions of the breast. Radiol Clin. North Am. 1983; 21:67-80.

14. Paulus, D.D. Benign disease of the breast. Radiol Clin North Amer. 1983; 21:27-50.

15. Bennett, I.C., Khan, A.. De Freitas, R., Chaudury," M.A. and Millis. R.R. Phyllodes tumours: a clinicopathological review of 30 cases. Aust. N. Z. J. Surg. 1992; 62:628-633.

16. Orel, S.G., Schnall, M.D., Li Volsi, V.A. and Troupin, R.T. Suspicious breast lesions: MR imaging with radiologicpathologic correlation. Radiology. 1994; 190:485-493.

17. Heywang-Kobrunner, S.H. Contrast cnhanced magnetic resonance imaging of the breast. Invest. Radiol. 1994; 29:94-104.

18. Hart. W.R., Bauer, R. and Oberman, H.A. Cystosatcoma phyllodes: a clinicopathological study of 26 hypercellular periductal stromal tumors of the breast. Amer, J. Clin. Pathol. 1978: 70:211-216.

19. Palmer, M.L., DeRisi, D.C., Pelikan. A., Patel, J. and Dao, T.L. Treatment options and recurrence potential for cystosarcoma phyllodes. Surg. Gynecol. Obstet. 1990; 170:193-196.

20. Norris, H.J. and Taylor, H.B. Relationship of histological features to behaviour of cystosarcoma phyllodes: Analysis of ninety-four cases. Cancer. 1967; 20:2090-2099.

21. Ward R. M. and Evans H.L. Cystosarcoma phyllodes: A clinico-pathological study of 26 cases. Cancer. 1986; 58:2282-2289.

22. Mindikoslu. A. N. and Aktan K. Recurrent cystosarcoma phyllodes of breast: Full thickness excision of chest wall with immediate repair using stecl mesh and a latissimus dorsi myocutaneous flap. Brit. J. Surg. 1983; 36:519-521

23. Stockdalc A. D. and Lcader M. Phyllodes tumour of the breast: response to radiotherapy. Clin. Radiol. 1987; 38:287-288. 